\title{
Cutaneous Pseudolymphoma: A Brief Review and Report of a Case Treatment Using Rituximab
}

\author{
Akbar Mokhtarpour Neilagh1*, Mahdi Aghazadeh Barenji1, Shahin Behrouz Sharif ${ }^{2}$ \\ ${ }^{1}$ Department of Dermatology, School of Medicine, Urmia University of Medical Sciences, Iran \\ ${ }^{2}$ Department of Medical Genetic, Faculty of Medicine, Tabriz University of Medical Sciences, Iran
}

\begin{abstract}
Cutaneous pseudolymphoma (C-PSL) is characterized by heterogenous lymphoproliferative processes contributing to skin lesions which histologically and clinically mimic malignant cutaneous lymphomas. It is of most importance to diagnose such case precisely and discriminate them from malignant lymphomas since treatment protocols are distinctive. Standard medical approaches for C-PSL includes elimination of causative factors, topical, intralesional and systemic steroids, hydroxychloroquine, and photodynamic therapy. Rituximab as an anti CD20 monoclonal antibody was used in this study to treat a patient with 8 years clinical features of pseudolymphoma with no positive response to routine medications. Follow up of almost more than 18 months revealed a successful treatment without noticeable side effects or disease recurrence.
\end{abstract}

Keywords: Cutaneous Pseudolymphoma, Cutaneous Lymphoid Hyperplasia, Rituximab

\section{Introduction}

Being described for the first time by Kaposi (1891), cutaneous pseudolymphoma (C-PSL) or cutaneous lymphoid hyperplasia (CLH) is a condition characterized by localized or disseminated benign reactive polyclonal either T-cell or B-cell proliferation in reaction to several known or unknown stimuli. It refers to skin lesions and heterogeneous lymphoproliferative processes that histologically and/or clinically resemble malignant cutaneous lymphomas most of the time. Its clinical manifestations vary from tumor-like nodes, solitary livid nodules, plaques to flat cell infiltrates, which can be singular or multiple, lasting from weeks to years [1]. Although its etiology is unknown, the process includes accumulation of lymphocytes on the grounds of exposure to various unknown and known stimuli such as arthropod bite, infection, drugs (such as anti-histaminic and phenytoin vaccination), hyper-sensitization to injection, tattoo, trauma, chemical and physical agents, and Borrelia burgdorferi infection. Long-term antigenic stimulation, exposure to gold and nickel, HIV infections, and post zoster phenomenon are other implicated causes of PSL. The predominant involvement site in PSL is face and scalp [2].

Differentiation between PSL and true lymphoma is often difficult and tricky due to their almost similar clinical and pathological aspects and thus differentiation must always be based on a combination of clinical and histological data and also the use of immunohistochemistry and molecular biology techniques; considering their completely distinct prognosis and treatment, differentiation will be fundamental. The diagnosis of malignant lymphoma is established when three criteria are fulfilled: (I) exclusive presence or marked preponderance of medium-sized or larger lymphocytes, (II) absence of preferential involvement of the upper dermis, and (III) absence of extensive epidermal infiltration. Lesions are classified as pseudolymphoma when they meet the following criteria: (A) germinal centers, (B) marked paucity of medium-sized lymphocytes, and [C] sharp margination between adjacent groups of small and large lymphocytes. Unfortunately, not every histologic presentation fails neatly into this system of classification [3].

\section{Case Report}

Here we report a 38 years old female patient diagnosed as pseudolymphoma case for 8 years, who represented papules, plaques, and dense erythematous nodules in whole face, chick, and forehead in clinical skin examination. She also had diffused lesions on her both forearms. Lesions were severely itchy.

Medical history of patient demonstrated no record of specific drug consumption, tattoo, vaccination, and implantation. During past 8 years of complication, almost every routine medication concerning pseudolymphoma including systemic, topical, and intralesional Cortone injection, Hydroxychloroquine tablet, and even drugs such as Cyclosporine and Azathioprine were used on the grounds of resistance lesions.

Microscopic pathological analysis was done on face skin biopsies indicating skin tissue with nodular infiltration of 
lymphocytes and histiocytes centered in upper and mid dermis with mild extension around follicles. No lichenoid infiltration and basal vacuolar degeneration were seen. Overlying epidermis was intact. Background of upper dermal fibrosis was also seen. Diagnosis was cutaneous pseudolymphomatous infiltration. Pseudolymphomas including pseudolymphomatous drug reaction, allergic contact dermatitis and lymphocytoma cutis were suggested. It was recommended to notice clinicopathological correlations. The specimen was also under processed for Immunohistochemistry study. The IHC study revealed CD3, CD4, and CD8 positive in some dermal lymphocytes whereas CD20 was positive in all dermal lymphocytes. Diagnosis based on IHC report was reactive lymphocytic infiltration, mixed $\mathrm{B}$ cell and $\mathrm{T}$ cell type. Direct immunofluorescence (DIF) for anti-total immunoglobulin analysis showed negative status for anti-IgG, anti-C3, anti-
IgA, anti-IgM, and anti-fibrinogen. DIF conclusion was negative.

Taking into account the disease resistance in this particular patient and DIF result indicating CD20+ status, Rituximab as an anti CD20 medication was injected intravenously as following: $500 \mathrm{mg}$ IV injection per week lasting four weeks and an injection of $1 \mathrm{gr}$ IV three months later. Considerable rehabilitation was seen in the patients lesions a month after injection of the first dose.

Almost all the lesions were disappeared three months later as illustrated in fig. 1 .

The patient was examined the following one, three, six, and twelve months that no recurrence was observed. She has been also examined after 18 months and still there was not any sign of recurrence. There was only compliance over occasional itchy state of healed lesions.

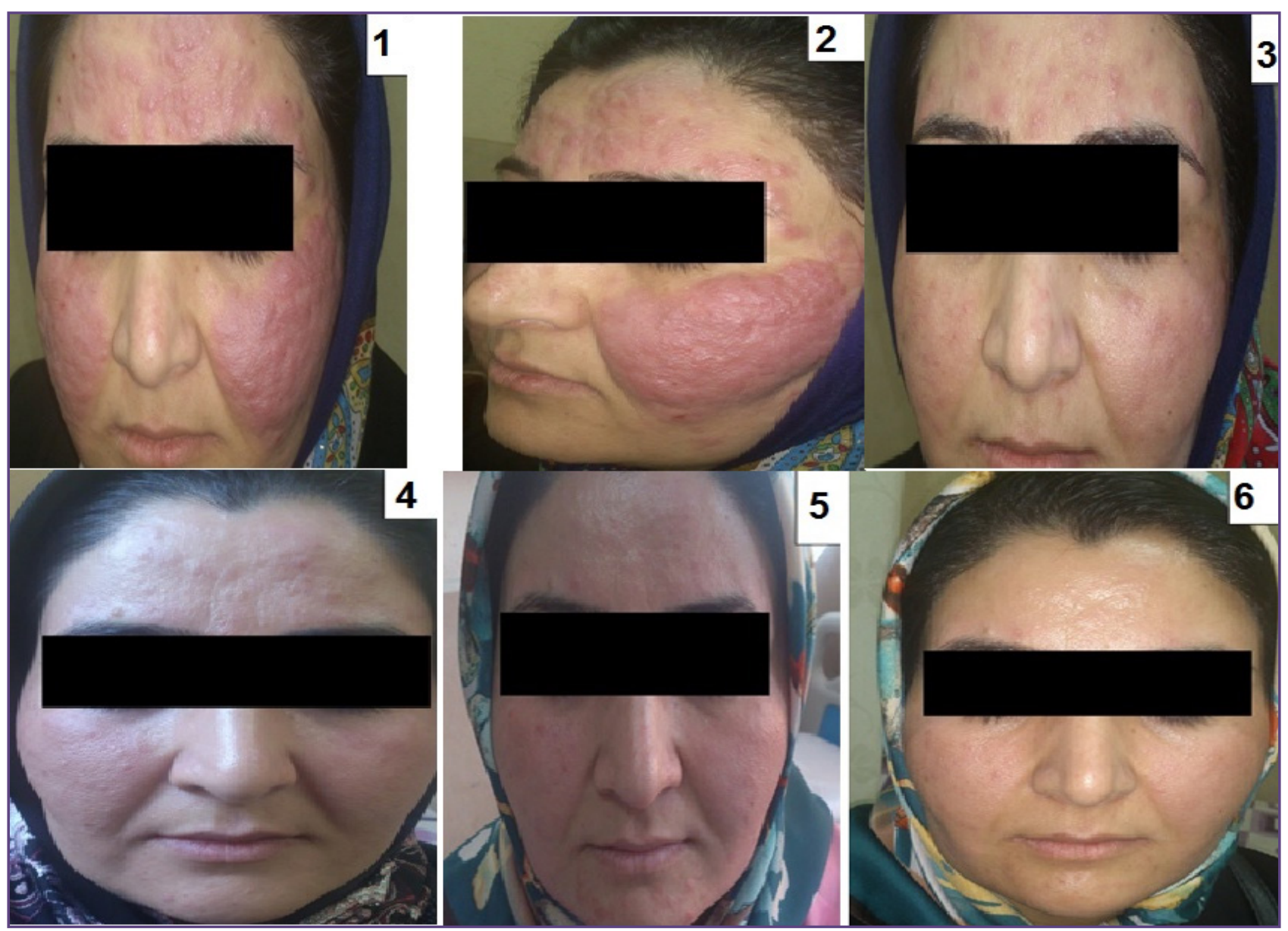

Fig. 1. Clinical manifestations of the case with cutaneous pseudolymphoma before and after treatment. These photos clearly indicate dramatic ceasation of cutaneous infiltrations which is the result of rituximab therapy. A and B indicate facial manifestations befor rituximab treatment. C represents one month after treatment. D represents three months after treatment. $\mathrm{E}$ and $\mathrm{F}$ represent six and twelve months after treatment, respectively. Comparing A and F, considerable healing of facial lessions can be noticed. 


\section{Discussion}

Treatment options for pseudolymphomas include excision, topical steroids, intralesional steroids, antibiotic, tacrolimus, systemic steroids, hydroxychloroquine, and photodynamic therapy. Therapy is mainly based on targeting the casual factor, for instance, withdrawal of causative drug or administering anti-parasitic medication in the case of persistent insect bite [4]. In a study by Dragonetti et al in 2004 a patient with cutaneous pseudolymphomas was successfully treated with hydroxychloroquine sulfate [400 $\mathrm{mg} / \mathrm{die}$ for three months] as the definite cause was not found. Administration of hydroxychloroquine sulfate was based on the fact that it has immunosuppressive activity and has successfully been used for the treatment of lupus erythematous [4]. Martin and Duvic reported a CLH case treatment with intralesional rituximab in 2011. The reported case was 58-year-old woman with a history of 18 months of slowly growing plaque on mid forehead and central scalp. They suggested that rituximab might be reasonable therapeutic option for CLH treatment [5]. Another report of case treatment with rituximab was stablished in 2010 by H. H. Ho et al. who managed to treat two cases with benign lymphoid hyperplasia of the orbit using rituximab. They also suggested no apparent side effects regarding rituximab therapy [6].

\section{Rituximab: mechanisms of action}

Despite of being a major choice for therapy strategies of a broad spectrum of B-cell malignancies and its undeniable therapeutic values, precise functional mechanism of rituximab as a monoclonal $\mathrm{IgG}$ is not yet fully understood [6]. Anti-cancer monoclonal antibodies are known to induce their effects by various mechanisms including cell cycle arrest by signaling induced apoptosis, complement dependent cytotoxicity (CMC) and antibody dependent cellular cytotoxicity (ADCC). Utilizing rapidly dividing tumor cell lines which are selected due to their in vitro rapid growth and sensitivity to therapy is one of the most common strategies in studying rituximab mechanisms of action. Although using animal models, which usually include mice with malignant cell lines represents more similar clinical situations, there are significant limitations. Differences in growth kinetics, phenotype, infiltration, and heterogeneity between mice tumors and clinical lymphoma, experimental situations, utilization of immune compromised animals and precise detection of therapeutic responses are major factors affecting our ability to relate animal responses to clinical mechanisms. On the other hand, clinical trials are valuable due to the measurement of events happening in patients although the majority of them pursue the assessment of therapeutic efficacy rather than understanding of mechanisms of action [7].
It has been shown that malignant B-cell line death can be induced by rituximab in vitro when the immune effector mechanisms are absent. Rituximab can induce changes in inhibition of p38 mitogen-activated protein kinase, NF$\mathrm{kB}$, ERK1/2 and AKT anti-apoptotic survival pathways [8]. Animal model studies have demonstrated synergic effects of rituximab and chemotherapy where prolonged exposure of rituximab to malignant cells can be studied in a physiologic environment. Moreover, rituximab has been reported in a study to have local anti-lymphoma effects when directly injected to cerebrospinal fluid of patients affected by CNS lymphoma [9].

Several studies, from in vitro analysis to correlative studies, have shown that complement dependent cytotoxicity (CMC) can be mediated efficiently by rituximab in fresh malignant samples and various B-cell lines [10]. Elimination of complement in tumor models abolishes effective therapeutic responses whereas cellular effector depletion has no influence on rituximab therapeutic responses. Moreover, complement activation during rituximab treatment and correlation of infusional toxicity with complement activation have been demonstrated in clinical observations [11].

It has been shown that ADCC of human lymphoma cells can be induced by rituximab using mononuclear cells. Furthermore, in animal models where CMC is not the central mechanism of rituximab therapy, it has been shown that ADCC plays the most important role. The association between CD16 gene polymorphisms and results of rituximab therapy suggests convincing evidences of ADCC involvement in responses to rituximab therapy. Better clinical responses in patients with follicular lymphoma with VV genotype have been demonstrated comparing to patients with VF or FF genotypes [12]. The importance of Fc-FcyR interactions are illuminated by these data suggesting ADCC as a major mechanism of action for rituximab.

Abovementioned points reveal independent major mechanisms of action for rituximab, however, several studies propose combination of these mechanisms to play a role in therapeutic pathways of a single model. Increased expression of anti-apoptotic and complement inhibitory molecules and decreased expression of CD20 can simultaneously contribute to rituximab responses. Extensive synergic or antagonistic interactions are also observed between these mechanisms [13].

In the present study, considering CD20+ status in all of infiltrated cells of the studies subject, rituximab was used as an anti-CD20 antibody. The possible interaction of rituximab Fc with $\mathrm{CD} 16$ and following ADCC might be 
the major treatment scenario in this case. Strong evidences of such interactions in rituximab mechanisms of action have culminated with the development of novel strategies for NK cell mediated cell lysis like development of next generation anti-CD20 antibodies with robust affinity for CD16 [14]. It has also been suggested by indirect evidences that regression of $\mathrm{B}$ cell lymphoma independent of strong immune participation can be induced by anti-CD20 therapy. Contribution of $\mathrm{CMC}$ to the clinical efficacy of rituximab treatment also has encouraged development of anti-CD20 antibodies with increased complement fixation properties [15].

\section{Conclusion}

Here, we report a successful treatment of a complicated pseudolymphoma case using rituximab. Although the exact mechanism of action is not clearly known, in any case of action such as altering signal transduction, $\mathrm{CMC}$, and ADCC, rituximab ca be assumed effective medication in pseudolymphoma treatment strategies, considering the results of this study. In addition to successful treatment, any serious side effects of rituximab were not present in our case indicating generally safe treatment protocol.

\section{Acknowledgements}

The authors want to sincerely thank the individual who contributed in this study as a case and authorized us to reveal these data including her medical complications and photos.

\section{Competing Interests}

There is not any declared conflict of interests between authors. Written informed consent is obtained from every individual participating in this study. This study is complient with ethical guidlines of human studies and approved by ethical committee of Urmia University of Medical Sciences.

\section{Reference}

1. Sequeira F, Jayaseelan E, Stephen J. (2014 Paraphenylenediamine-induced cutaneous pseudolymphoma. IJD. 2014;59[4]:424.

2. Nnebe NV, Woon C, Haines S, Dayton V, Weigel BJ. Cutaneous pseudolymphoma: an unusual presentation of a scalp mass. Pediatric blood \& cancer. 2009;52[2]:283-5.

3. Stoll DM. Treatment of cutaneous pseudolymphoma with hydroxychloroquine. Journal of the American Academy of Dermatology. 1983;8[5]:696-9.
4. Dragonetti E, Cianchini G, Mastrangelo L, Mellone P, Baldi A. Cutaneus pseudolymphoma: a case report. in vivo. 2004;18[5]:549-52.

5. Martin SJ, Duvic M. Treatment of cutaneous lymphoid hyperplasia with the monoclonal anti-CD20 antibody rituximab. Clinical Lymphoma Myeloma and Leukemia. 2011;11[3]:286-8.

6. McLaughlin P, Grillo-López AJ, Link BK, Levy R, Czuczman MS, Williams ME, et al. Rituximab chimeric antiCD20 monoclonal antibody therapy for relapsed indolent lymphoma: half of patients respond to a four-dose treatment program. Journal of clinical oncology. 1998;16[8]:2825-33.

7. Weiner GJ, editor. Rituximab: mechanism of action. Seminars in hematology; 2010: Elsevier.

8. Czuczman MS, Olejniczak S, Gowda A, Kotowski A, Binder A, Kaur H, et al. Acquirement of rituximab resistance in lymphoma cell lines is associated with both global CD20 gene and protein down-regulation regulated at the pretranscriptional and posttranscriptional levels. Clinical Cancer Research. 2008;14[5]:1561-70.

9. Harjunpaa A, Junnikkala S, Meri S. Rituximab [anti-CD20] therapy of B-cell lymphomas: direct complement killing is superior to cellular effector mechanisms. Scandinavian journal of immunology. 2000;51[6]:634.

10. Reff ME, Carner K, Chambers K, Chinn P, Leonard J, Raab R, et al. Depletion of B cells in vivo by a chimeric mouse human monoclonal antibody to CD20. Blood. 1994;83[2]:435-45.

11. Van der Kolk L, Grillo-López A, Baars J, Hack C, Van Oers M. Complement activation plays a key role in the side-effects of rituximab treatment. British journal of haematology. 2001;115[4]:807-11.

12. Weng W-K, Levy R. Two immunoglobulin G fragment $\mathrm{C}$ receptor polymorphisms independently predict response to rituximab in patients with follicular lymphoma. Journal of clinical oncology. 2003;21[21]:3940-7.

13. Dalle S, Dupire S, Brunet-Manquat S, Reslan L, Plesa A, Dumontet C. In vivo model of follicular lymphoma resistant to rituximab. Clin. Cancer Res. h. 2009; $15[3]: 851-7$.

14. Robak T. GA-101, a third-generation, humanized and glyco-engineered anti-CD20 mAb for the treatment of B-cell lymphoid malignancies. Curr Opin Investig Drugs. 2009;10[6]:588-96.

15. Klepfish A, Schattner A, Ghoti H, Rachmilewitz EA. Addition of fresh frozen plasma as a source of complement to rituximab in advanced chronic lymphocytic leukaemia. Lancet Oncol. 2007;8[4]:361-2.

*Corresponding author:

Akbar Mokhtarpour Neilagh, Orjhans Street, Resalt Blvd, Urmia, Iran, 571478334

Phone: +919171060599

Email: mokhtarpour.akbar@gmail.com

Financial or other Competing Interests: None. 